\title{
First report on soapnut extract-mediated synthesis of sulphur- substituted nanoscale NdFeB permanent magnets and their characterization
}

\author{
G. V. S. Jayapala Rao ${ }^{1}$ T. N. V. K. V. Prasad ${ }^{2} \cdot$ Syed Shameer $^{2} \cdot$ \\ T. Arun $^{3} \cdot$ M. Purnachandra Rao ${ }^{4}$
}

Received: 8 June 2017/ Accepted: 19 August 2017/Published online: 28 August 2017

(c) The Author(s) 2017. This article is an open access publication

\begin{abstract}
Biosynthesis of nanoscale materials has its own advantages over other physical and chemical methods. Using soapnut extract as reducing and stabilizing agent for the synthesis of inorganic nanoscale materials is novel and has not been exploited to its potential so far. Herein, we report for the first time on the effects of sulphur substitution on soapnut extract-mediated synthesis of nanoscale $\mathrm{NdFeB}$ $(\mathrm{S}-\mathrm{NdFeB})$ permanent magnetic powders $(\mathrm{Nd} \mathrm{15 \% ,} \mathrm{Fe}$ $77.5 \%$, B 7.5\% and $\mathrm{S}$ with molar ratios: $0.1,0.2,0.3,0.4$, and 0.5 ). To synthesize, a $10 \mathrm{ml}$ of $10 \%$ soapnut extract was added to $90 \mathrm{ml}$ of respective chemical composition and heated to $60{ }^{\circ} \mathrm{C}$ for $30 \mathrm{~min}$ and aged for $24 \mathrm{~h}$. The dried powder was sintered at $500{ }^{\circ} \mathrm{C}$ for $1 \mathrm{~h}$. The characterization of the as-prepared nanoscale $\mathrm{S}-\mathrm{NdFeB}$ magnetic materials was done using the techniques such as X-ray diffraction (XRD), scanning electron microscopy (SEM) with energy dispersion spectroscopy (EDS), Fourier transform infrared spectroscopy (FT-IR), dynamic light scattering (DLS for size and zeta potential measurements) and vibrating sample magnetometer (VSM)-hysteresis loop studies. The results revealed that particles were highly stable (with a negative zeta potential of $25.7 \mathrm{mV}$ ) with irregular and spherical
\end{abstract}

T. N. V. K. V. Prasad

tnvkvprasad@gmail.com

1 Department of Physics, Maharajas College (Autonomous), Vizianagaram, Andhra Pradesh, India

2 Nanotechnology Laboratory, Institute of Frontier Technology, Regional Agricultural Research Station, Acharya N G Ranga Agricultural University, Tirupati, Andhra Pradesh, India

3 Institute of Physics, Bhubaneswar, India

4 Department of Physics, Andhra University, Visakhapatnam, Andhra Pradesh, India shape (with measured hydrodynamic diameter 6.7 and $63.5 \mathrm{~nm}$ ). The tetragonal structures of the formed powders were revealed by XRD micrographs. Hysteresis loop studies clearly indicate the effect of $\mathrm{S}$ concentration on the enhanced magnetization of the materials.

Keywords Soapnut - NdFeB - Ferromagnetism ·

Biosynthesis · Sulphur · Hysteresis loop

\section{Introduction}

Magnets have been fascinating mankind since ancient times. There are a number of fields in which magnets are being used including acoustics, heavy electrical devices, data storage, and devices used for imaging through magnetization. Over ages iron-based magnetic materials have been attracting the scientific community due to their unique characteristics such as large remanence magnetization, high-energy density, and enhanced coercive force. Among iron-based ferromagnetic systems, $\mathrm{NdFeB}$ system (with highest energy product $\mathrm{BH}$ max) was proved to be one of the prominent ferromagnetic systems whose production is under exploitation (Brown et al. 2002) and possesses remanence of up to $1.45 \mathrm{~T}$ and a maximum coercivity of $2650 \mathrm{kA} / \mathrm{m}$ (Chopra et al. 2007).

Nowadays, nanoscale materials (size $<100 \mathrm{~nm}$ in at least one dimension) of all kinds have been told to be the new-generation materials including magnetic materials. There are notable differences in physical, chemical and biological properties exhibited by nanoscale materials compared to their bulk counterparts. Most importantly, at nanoscale, the surface area of the material per unit volume increases to several folds, which makes them more suitable for high-end applications like medicine, space 
research, etc. However, the magnetic properties of nanoscale materials were determined by their chemical composition, methods employed for synthesis, crystalline lattice, crystalline defectiveness, particle size, shape and surface morphology (Zeng and sun 2008; Rao and Cheetham 2011). As the demand of $\mathrm{NdFeB}$ magnets has been increasing rapidly in various fields of life science due to their excellent magnetic performance, it is required to develop low-cost and eco-friendly synthetic routes to produce $\mathrm{NdFeB}$ magnets.

Various methods were employed (Shah et al. 2015) to synthesize $\mathrm{NdFeB}$ ferromagnetic materials alone and with co-substitution including melt spinning, atomization (Liu and Zhao 2017), hydrogen disproportionate desorption recombination (HDDR) (Gutfleisch et al. 2002) and mechanically alloying to achieve enhanced magnetization (Deheri et al. 2010). However, the methods of preparation involve high-end chemicals with the lengthy procedures with required skills (Jadhav et al. 2014)

Biosynthesis of nanoscale materials using various biological entities (microbes, plants, and bio-templates) (Madhavi et al. 2013; Kotakadi et al. 2014) has been developing as a special branch of nanobiotechnology at a rapid pace. Using plant and plant materials for the synthesis of inorganic nanoscale materials has advantages over using other biological entities (Prasad et al. 2011; Sindhura et al. 2014) in the synthesis protocol. Through biological methods, with minimum energy using eco-friendly synthetic routes, nanoscale materials could be produced (Elumalai et al. 2011). Soapnut fruit is popular in Asia and its extract has been used as a surfactant over ages. The fruit extract is also used to inhibit tumour cells and exhibits contraceptive activity (George and Shanmugam 2014). Soapnut fruit extract contains various phenolic compounds, flavonoids, and carbohydrates. Bharti et al. (2012) argue as to recognize this extract as one of the potential candidates for the synthesis of metallic nanoparticles and compositions. However, reports on the use of soapnut extract for synthesizing metallic nanoparticles are scarce. In the present investigation, we report for the first time on the synthesis of $\mathrm{NdFeB}$ ferromagnetic materials using soapnut extract as reducing and stabilizing agent and also we have evaluated the effect of sulphur substitution against boron on the structure and magnetic properties of $\mathrm{NdFeB}$ permanent magnets.

\section{Experimental}

\section{Materials}

Neodymium (III) nitrate hexahydrate $\left[\mathrm{Nd}\left(\mathrm{NO}_{3}\right)_{3} \cdot 6 \mathrm{H}_{2} \mathrm{O}\right.$, 99.9\%, Aldrich Chemicals], ferric nitrate anhydrous (extra pure, AR, ACS) $\left(\mathrm{Fe}\left(\mathrm{NO}_{3}\right)_{3} \cdot 9 \mathrm{H}_{2} \mathrm{O}\right)$, boric acid (extra pure, $\mathrm{AR}, \mathrm{SRL}, \mathrm{H}_{3} \cdot \mathrm{BO}_{3}$ ), and sulphur powder (HIMEDIA) were used as received without further purification and doubledistilled water (DDW) as solvent. Soapnuts (Dried, $100 \mathrm{~g}$ ) purchased from the local market in Tirupati, Andhra Pradesh, India and used as received.

\section{Methods}

Dried soapnuts $(100 \mathrm{~g})$ were milled to get fine powder using a grinder. Then $10 \mathrm{~g}$ of fine soapnut powder was added to $100 \mathrm{ml}$ of distilled water (DW) and heated to $80{ }^{\circ} \mathrm{C}$ for $30 \mathrm{~min}$. After heating, the solution was filtered using filter paper (Whatman no. 1) and an aliquot (10\%) was collected for further experimentation.

\section{Preparation of soapnut extract-mediated S-NdFeB magnetic systems}

The precursors of metal nitrates $\mathrm{Nd}\left(\mathrm{NO}_{3}\right)_{3} \cdot 6 \mathrm{H}_{2} \mathrm{O}, \mathrm{Fe}$ $\left(\mathrm{NO}_{3}\right)_{3} \cdot 9 \mathrm{H}_{2} \mathrm{O}$ and boric acid were taken in the volumetric ratios of $\mathrm{Nd}: \mathrm{Fe}: \mathrm{B}-15: 77.5: 7.5$ for the synthesis of $\mathrm{Nd}_{2}$ $\mathrm{Fe}_{14} \mathrm{~B}$ phase. To prepare the S-NdFeB permanent magnetic system, $10 \mathrm{ml}$ of $10 \%$ of soapnut extract was thoroughly mixed with the mixture of 1 molar concentration of individual elements at 15:77:7.5 volumetric ratios of $\mathrm{Nd}, \mathrm{Fe}$, and $\mathrm{B}$, respectively, with the substitution of sulphur at a variation of $0.1,0.2,0.3,0.4$, and 0.5 in the proportion of Boron (B) cumulating to no overall change in the ratios. The mixture was heated to $60{ }^{\circ} \mathrm{C}$ and cooled to room temperature, and further aged at room temperature for $24 \mathrm{~h}$. The precipitated S-NdFeB magnetic particles were separated from the mixture through ultrafiltration and dried. The S-NdFeB magnetic powders were sintered at $500{ }^{\circ} \mathrm{C}$ for $1 \mathrm{~h}$. The formed nanoscale $\mathrm{S}-\mathrm{NdFeB}$ magnetic materials were stored for further characterization assays.

\section{Characterization of the prepared nanoscale S-NdFeB magnetic powders}

\section{Dynamic light scattering spectroscopy (DLS for size and zeta potential measurements)}

Dynamic light scattering (DLS) technique was employed using a particle sizing system [Nanopartica-SZ-100 (HORIBA)] at a wavelength of $633 \mathrm{~nm}$ from a $4.0 \mathrm{~mW}$, solid-state $\mathrm{He}-\mathrm{Ne}$ laser at a scattering angle of $170^{\circ}$. The hydrodynamic diameter (size) was measured from built-in software for all the samples prepared (Takahashi et al. 2008). 


\section{X-ray diffraction (XRD)}

The XRD patterns of prepared samples were taken using an $\mathrm{X}$-ray diffractometer (Rigaku) operating in the reflection mode with $\mathrm{CuK} \alpha$ radiation $(k=0.1542 \mathrm{~nm}$ ). The scanning range was between $2 \theta=20-80$ with a step size of 0.04 .

\section{Scanning electron microscopy (SEM) and energy dispersion spectroscopy (EDS)}

Surface morphological details of the prepared nanoscale materials could be studied from SEM micrographs. The SEM (Carl Zeiss, Neon 40, 1.1 nm @ 20 kV, 4-20 nA) microscope was used in this study to reveal the surface morphology, size, and shape of the prepared samples.

\section{Fourier transform infrared spectroscopy (FT-IR)}

The functional groups present on the surface of powders and in solution were identified using Fourier transform infrared (FT-IR) spectroscopy (Perkin-Elmer) in the frequency range $4000-400 / \mathrm{cm}$ by pelletizing a homogenized powder of the synthesized particles and $\mathrm{KBr}$ (ratio 1:200). The spectra were corrected by subtracting the spectrum of a $\mathrm{KBr}$ blank pellet and were presented in the transmittance mode (Song et al. 2009).

\section{Vibrating sample magnetometer (VSM)-hysteresis loop studies}

A vibrating sample magnetometer (VSM) works on the principle of Faraday's law of electromagnetic induction. The field available in between electromagnets is $2 \mathrm{~T}$. The system is calibrated with a cylindrical $\mathrm{Ni}$ specimen of weight $0.0925 \mathrm{~g}$ at a uniform magnetic field of $5 \mathrm{kOe}$ to give a saturation moment of about $56 \mathrm{emu} / \mathrm{g}$ calibration, which could be maintained within $\pm 0.05 \%$. The frequency of vibration is $75 \mathrm{~Hz}$. Typically around $20-50 \mathrm{mg}$ of the composite powder sample is used for VSM study. The specimen is fastened in a non-magnetic holder of $3 \mathrm{~mm}$ diameter, which is vibrated at a frequency of $75 \mathrm{~Hz}$ in a uniform magnetic field generated by an electromagnetic field.

\section{Results and discussion}

\section{Biosynthesis of sulphur-substituted NdFeB ferromagnets}

Biosynthesis of metallic nanoparticles has advantages over conventional methods (chemical and physical methods) in terms of ease of procedure and attaining high purity of the prepared nanoscale materials. There are a number of reports available in the literature ( $\mathrm{Lu}$ et al. 2007; Thakkar et al. 2010) and, in all, synthesis of $\mathrm{NdFeB}$ magnetic materials involves high-end chemicals, critical procedures and requires high energy. Biosynthesis of materials, nanoscale materials in particular, has so many advantages over conventional chemical and physical methods in obtaining the desired materials (Elumalai et al. 2011). Also using plant products or plant extracts in the synthetic protocol has many advantages over their counterparts in terms of handling the precursors (Prasad et al. 2011). Soapnut extract (composition of a variety of functional groups including alcohols, thiols) (Sharma et al. 2011) has the ability to reduce and stabilize the materials during the respective processes and was successfully used in the present study to prepare nanoscale S-substituted $\mathrm{NdFeB}$ magnetic materials.

\section{Dynamic light scattering spectroscopy (DLS for size and zeta potential measurements)}

Dynamic light scattering (DLS) technique is used to measure the hydrodynamic diameter (HDD) of the particles. It is evident from Fig. $1 \mathrm{a}, \mathrm{b}$ that the formed magnetic nanoparticles have mean diameter of $6.7-63.5 \mathrm{~nm}$ and are uniformly distributed. The $1.5 \%$ substitution of sulphur was found to be the best concentration for the synthesis of sulphur-doped $\mathrm{NdFeB}$ nanomaterials. In addition, prepared magnetic nanoparticles were highly stabilized which is evident from the recorded zeta potentials (Fig. 2). Higher coercivity can be achieved by finer particles.

\section{X-ray diffraction (XRD)}

X-ray diffraction analysis was done for the confirmation of expected fabrication and determination of crystal structure of the prepared samples. X-ray diffraction patterns of S-substituted NdFeB system is shown in Fig. 3. For each peak, interplanar spacing ' $d$ ' was calculated using Bragg's law $(n \lambda=2 d \sin \theta)$ and was compared with interplanar spacing ' $d$ ' in JCPDS database. The X-ray diffraction patterns clearly indicate the Bragg's reflections from the

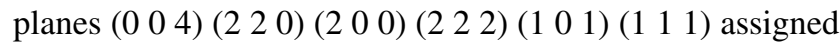
to a tetragonal crystalline structure. The phase identification and determination of crystallographic parameters are matched with the JCPDS 39-0473 assigned for $\mathrm{Nd}_{2} \mathrm{Fe}_{14} \mathrm{~B}$. It is evident from Fig. 3 that the c-axis is strongly oriented perpendicular to the plane of the crystal because the peaks such as 200 and 400 which are the reflections of $\varnothing$ phase have considerably high intensity (Tian et al. 2013). However, substitution of $\mathrm{S}$ does not affect the crystalline structure of $\mathrm{NdFeBs}$. 


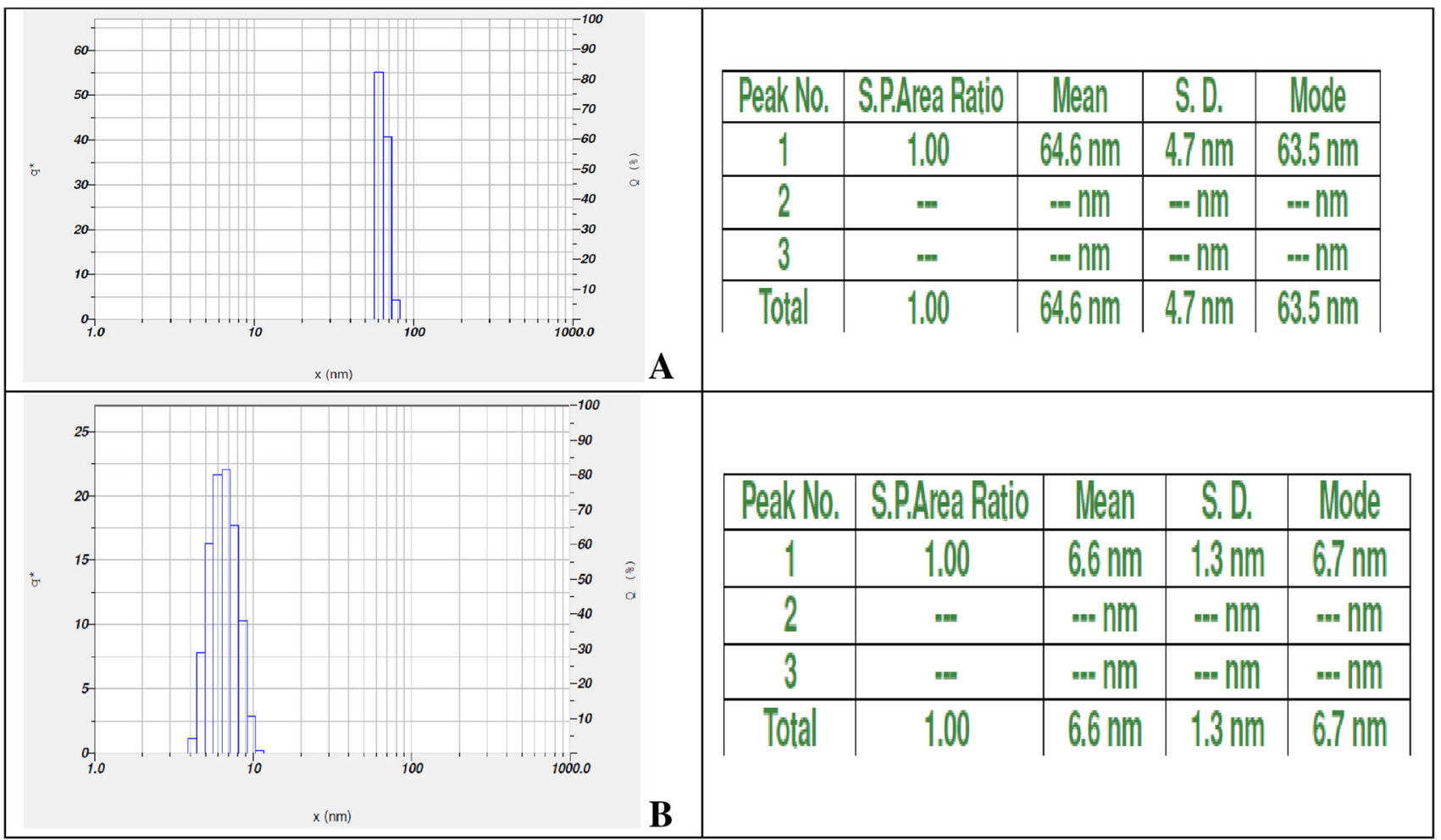

Fig. 1 Particle size distribution (using dynamic light scattering) of S-substituted NdFeB magnetic materials. a $0.2 \%$ S-substituted NdFeB. b $0.5 \%$ S-substituted $\mathrm{NdFeB}$

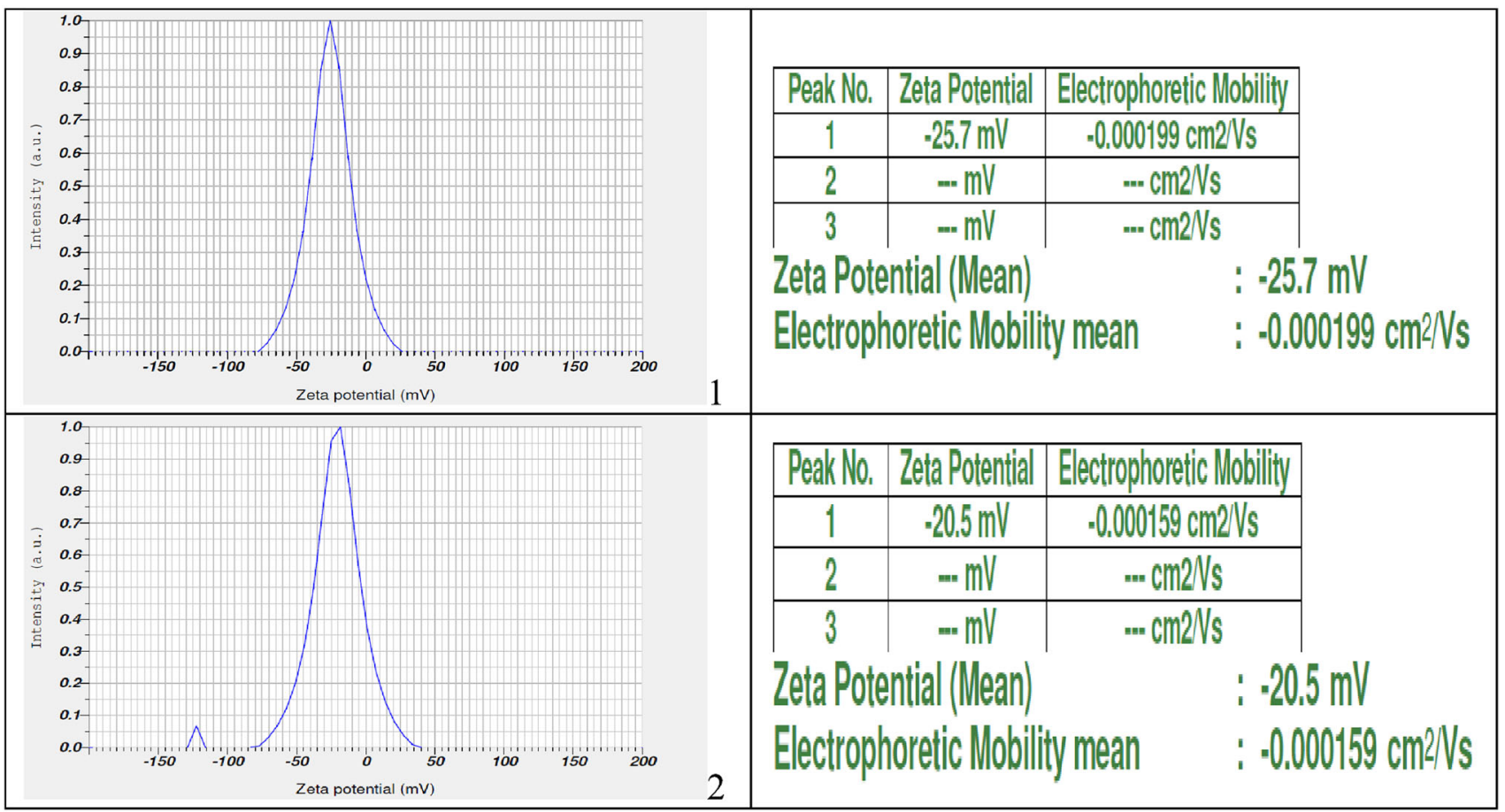

Fig. 2 Zeta potential of the synthesized S-substituted NdFeB magnetic nanoparticles. 1 S $0.2 \%$ NdFeB. 2 S $0.5 \%$ NdFeB 

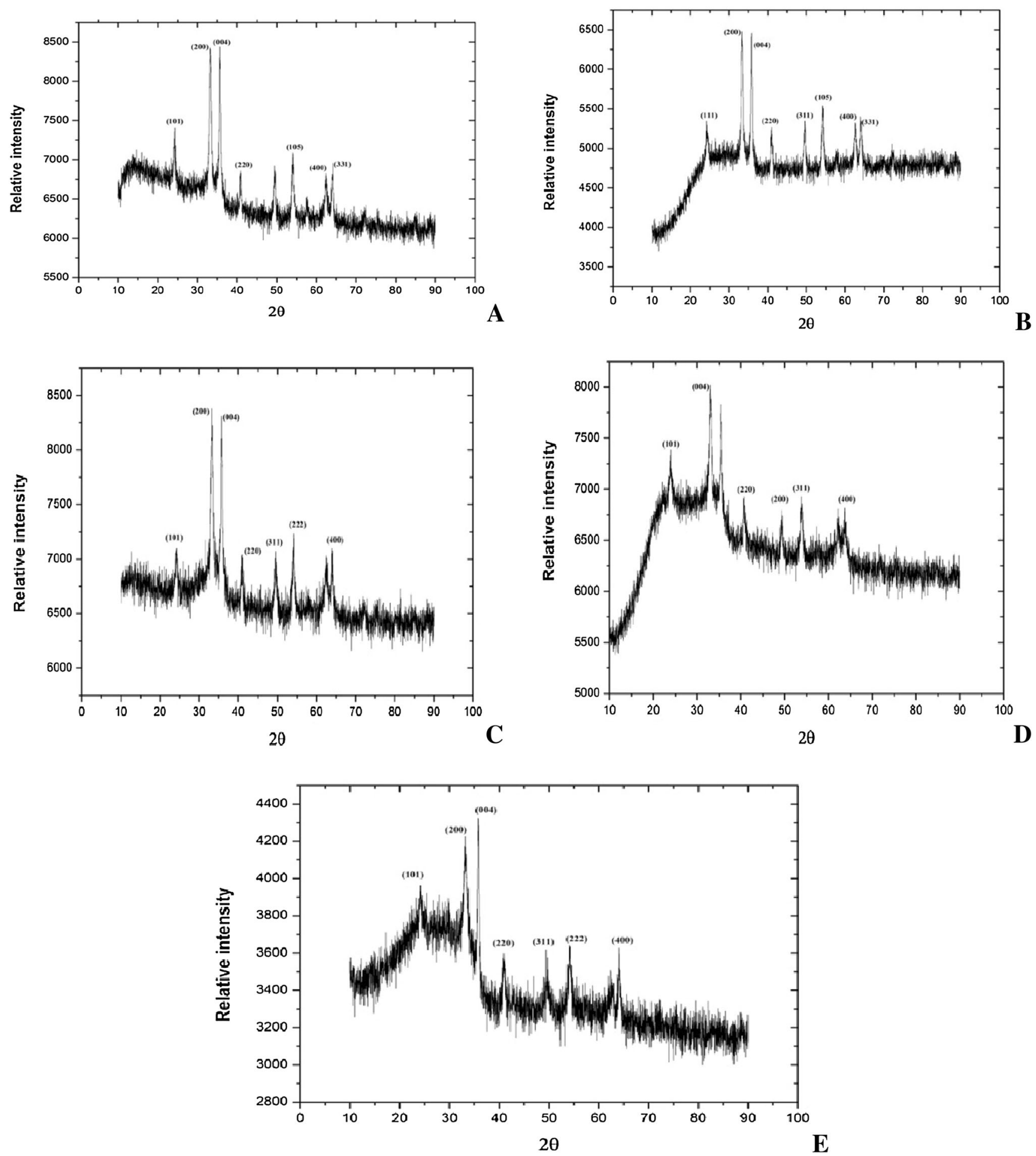

Fig. 3 X-ray diffraction micrographs representing the indexed Bragg's reflections of S-substituted NdFeB ferromagnetic materials. a 0S 0.2\% NdFeB. b S $0.3 \%$ NdFeB. c S $0.4 \%$ NdFeB. d S $0.5 \% \mathrm{NdFeB}$ and e S $0.0 \% \mathrm{NdFeB}$

Scanning electron microscopy (SEM) and energy dispersion spectroscopy (EDS)

The scanning electron microscopy of the synthesized $\mathrm{NdFeB}$ nanoparticles revealed the formation of $\mathrm{NdFeB}$ particles in different nanometer range and the substitution of sulphur in the proportion of iron varied the grain size and structure significantly. The grain sizes averaging in the range $6.7-63.5 \mathrm{~nm}$ were observed when the substitution concentration of $0.3 \%$ of sulphur in the core proportion of 

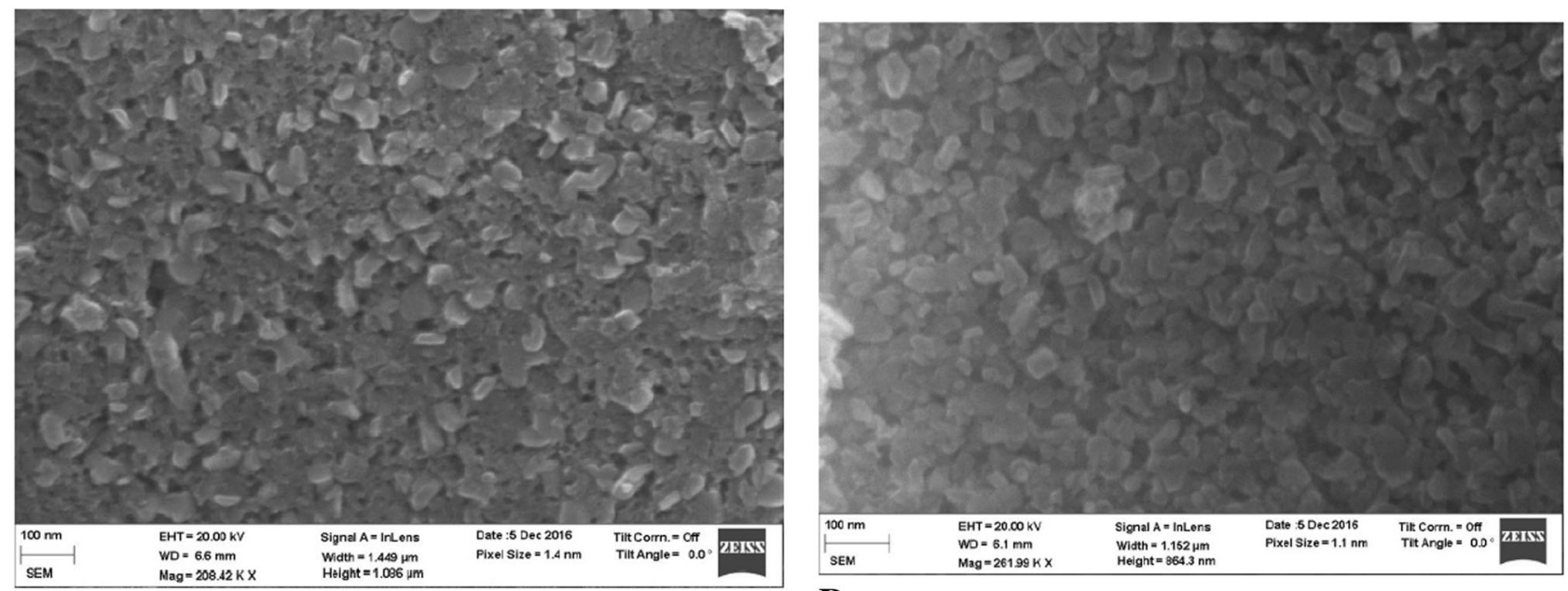

A

B

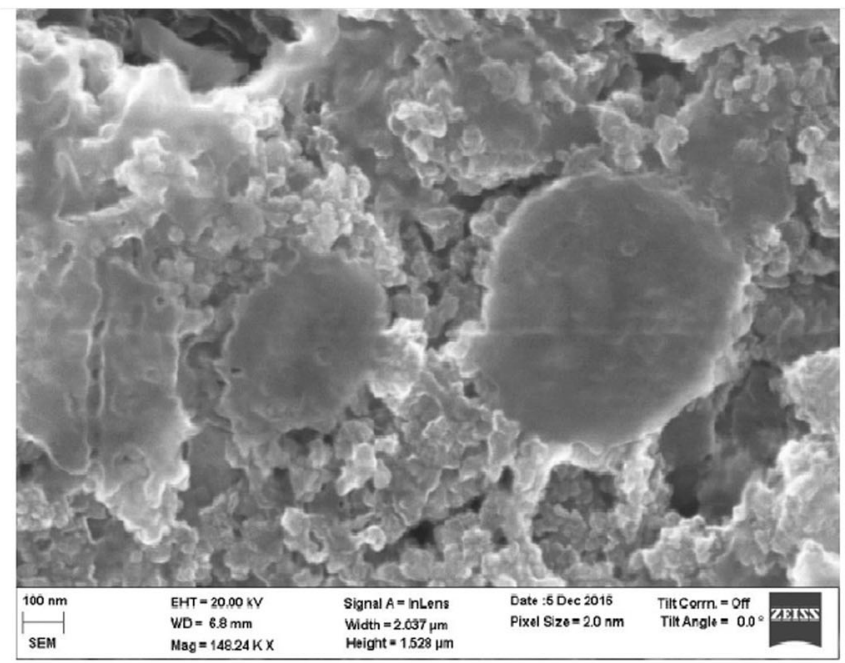

C

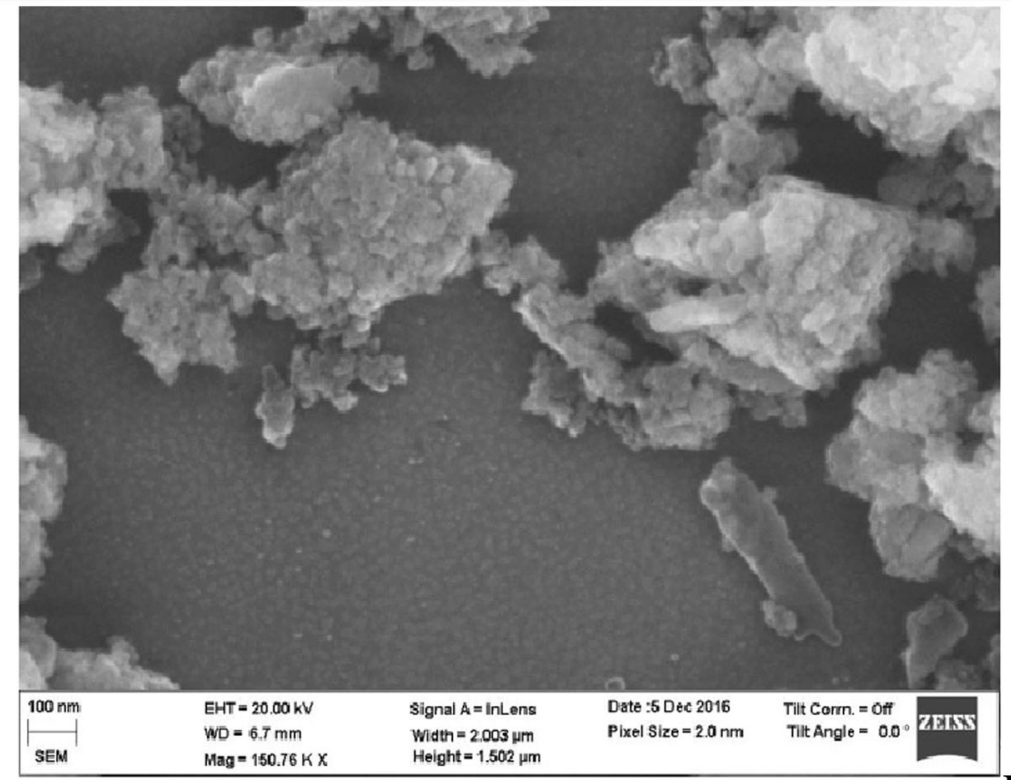


4Fig. 4 Scanning electron micrographs showing the surface morphology, size and shape of the S-substituted $\mathrm{NdFeB}$ ferromagnetic nanoparticles. a S $0.2 \% \mathrm{NdFeB}$. b S $0.3 \% \mathrm{NdFeB}$. c S $0.4 \% \mathrm{NdFeB}$. d S $0.5 \% \mathrm{NdFeB}$ and e $\mathrm{S} 0.0 \% \mathrm{NdFeB}$
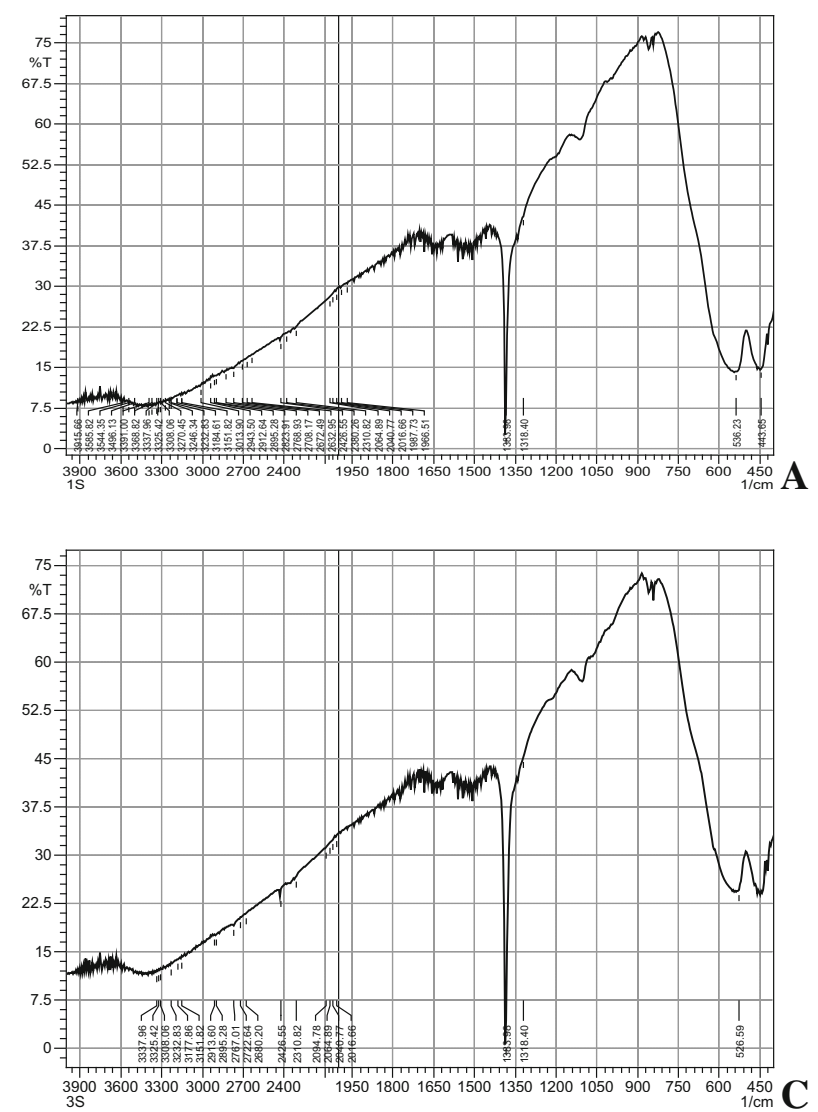

the crystal system was taken. As the substitution increased past $0.5 \%$, we observed the grain size also increased which can be accredited to stretch caused by the sulphur molecules in the crystal. The shape of the formed crystals also
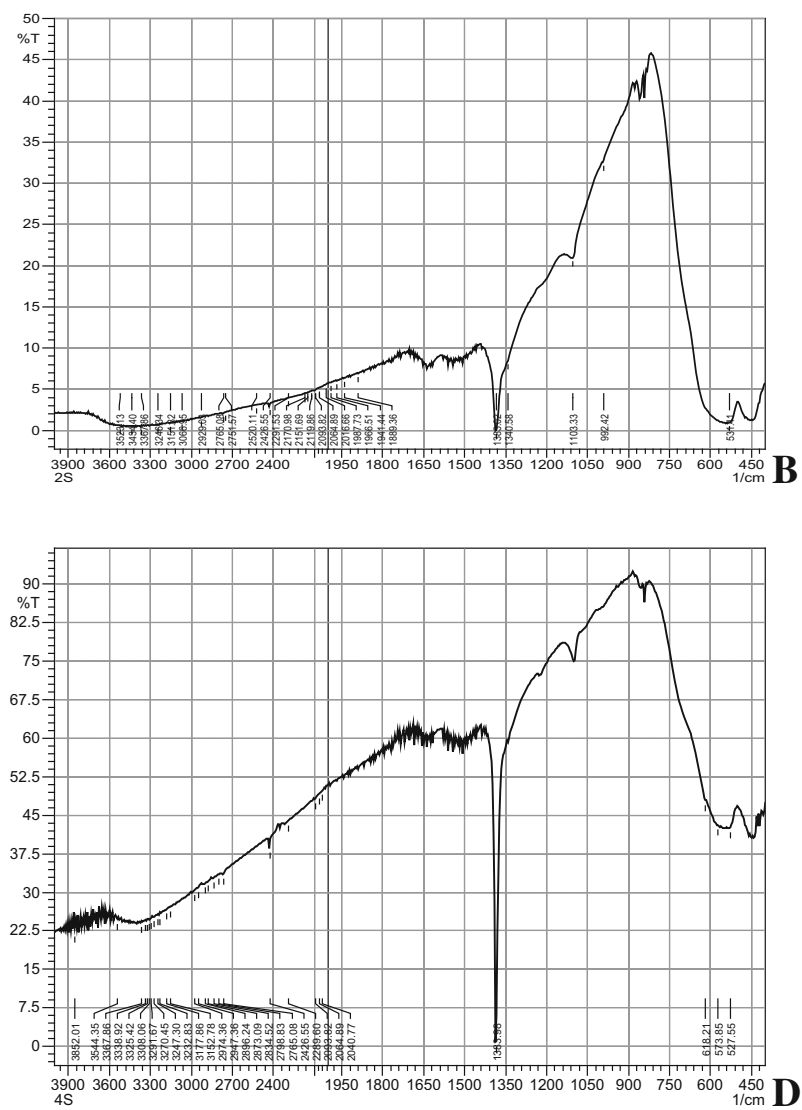

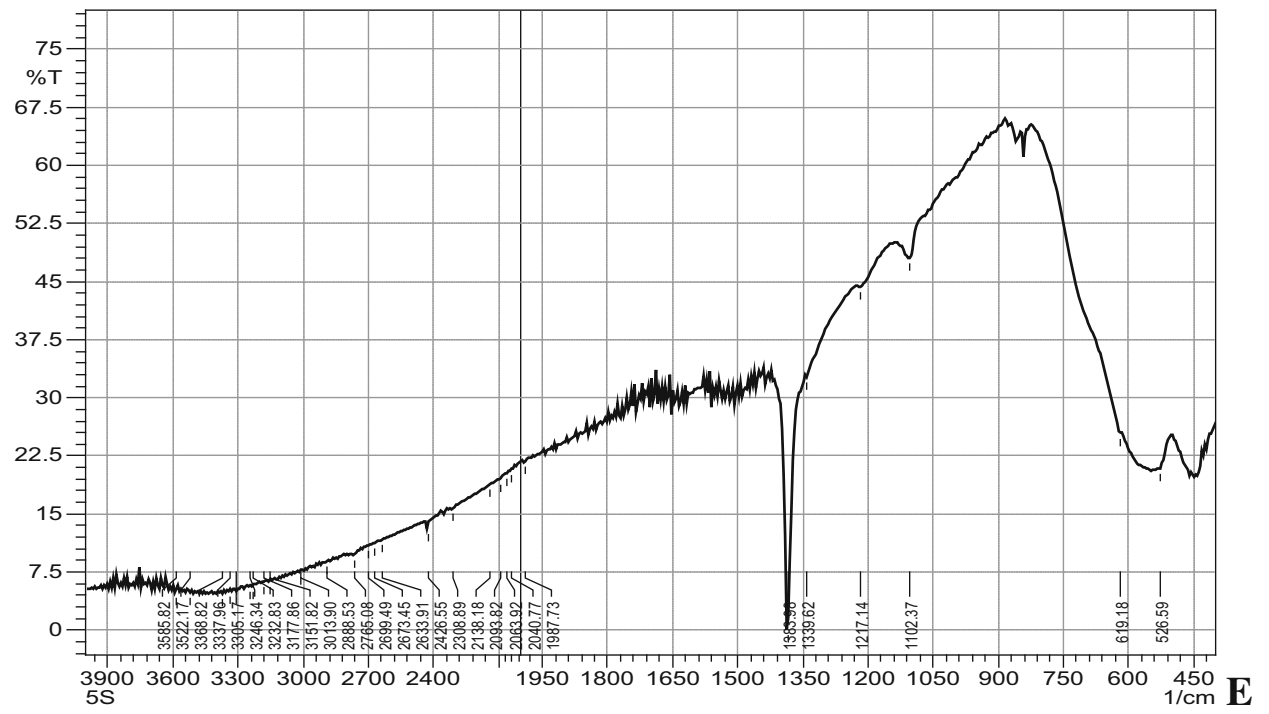

Fig. 5 FT-IR spectrums representing the functional groups involved in the reduction and stabilization processes and also their presence on the surfaces of the S-substituted $\mathrm{NdFeB}$ ferromagnetic nanoparticles. a $0 \mathrm{~S} 0.2 \% \mathrm{NdFeB}$. b S $0.3 \% \mathrm{NdFeB}$. c S $0.4 \% \mathrm{NdFeB}$. d S $0.5 \%$ $\mathrm{NdFeB}$ and e $\mathrm{S} 0.0 \% \mathrm{NdFeB}$ 
was recorded to be influenced by the sulphur doping (Castaldi et al. 2002). The SEM images also showed the different sulphur concentrations substitution in $\mathrm{NdFeB}$ system leads to formation of particles with different sizes and shapes which may find diverse applications. Concurrently, the energy-dispersive X-ray spectroscopy (EDS) revealed that the substitution of sulphur was successful but the peaks for the corresponding substitution may not be very distinct as the substitution is very low at about $0.5 \%$ only. The SEM images of the sulphur-doped NdFeB nanoparticles are shown in Fig. 4.

\section{Fourier transform infrared spectroscopy (FT-IR)}

The FT-IR analysis of the S-substituted NdFeB nanoparticles showed characteristic absorption peaks of different functional groups ranging from disulphides $(705-570 / \mathrm{cm})$, polysulphides (500-470/cm), cyanate (2260-2240/ $1190-1080 / \mathrm{cm})$, thiocyanate $(2175-2140 / \mathrm{cm})$, ammonium ion $(3300-3030 / 1430-0390 / \mathrm{cm})$, nitrate ion (1380-1350/840-815/cm), hydroxyl group (3570-3200 / $\mathrm{cm})$, polymeric O-H $(3400-3200 / \mathrm{cm})$, methoxy C-S stretch (2820-2810/cm), etc. (Wu et al. 2015). The FT-IR spectrum shows the substitution of $\mathrm{S}$ in the $\mathrm{NdFeB}$ system has greatly influenced the characteristic functional groups of the magnetic nanoparticles when compared with nonsubstituted control (Fig. 5). The number of distinct absorption peaks serves as a measure of the influence of sulphur doping which shows a dramatic increase in a number of peaks with an increase in sulphur concentration. The variations also in the presence of absorption peaks of the functional groups may be attributed to sulphur substitution; as the concentration of sulphur increased, functional groups also increased and their peak shapes fluctuated greatly from broad to sharp and, in some instances, the opposite was observed. The soapnut extract without any faltering gave rise to different functional groups; as a result, the bioapplicability of the $\mathrm{NdFeB}$ nanoparticles is greatly improved.

\section{Hysteresis loop studies}

Magnetic materials are renowned by their behaviour in external magnetic fields. For ferromagnets, the $M(H)$ dependence is characterized by domain wall movement and magnetization rotation. According to the theory of Weiss, a non-saturated ferromagnet contains a number of small regions called domains, in which the local magnetization is homogeneous and reaches the saturation value. Two domains are separated by domain walls which are regions of approximately $100 \mathrm{~nm}$ where the magnetization rotates continuously. The variation of magnetic flux is created by the vibration of the specimen in a DC magnetic field. The
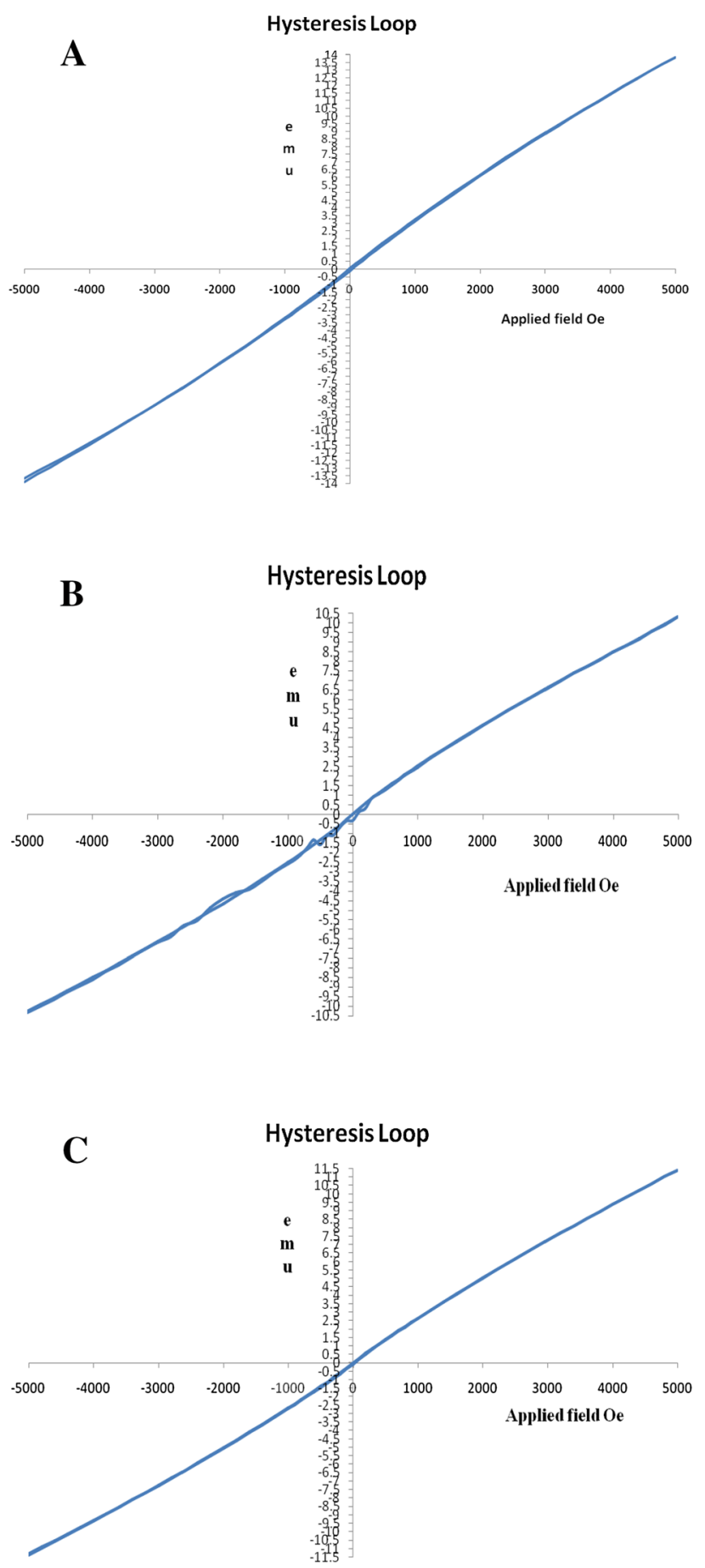

Fig. 6 Hysteresis loop studies of sulphur-substituted soapnut extractmediated $\mathrm{NdFeB}$ ferromagnetic materials. a $0 \%$ S-Substituted NdFeB. b $0.3 \%$ S-substituted NdFeB and c $0.5 \%$ S-substituted NdFeB

magnetization was measured by the pickup coils and direct interface dedicated computer was used for the data acquisition. The NdFeB nanoparticles synthesized with sulphur substitution at variation $0.3 \%$ in the core proportion of 15:77.5:[7.0 +0.5] (NdFeB's Boron) had coercive field $\left(H_{\mathrm{c}}\right)$ value of 36.539 , saturation magnetization $\left(M_{\mathrm{s}}\right)$ of 
$10.580 \mathrm{E}-3$, remanent magnetization $\left(M_{\mathrm{r}}\right)$ of $173.520 \mathrm{E}-6$, and saturation $\left(H_{\mathrm{s}}\right)$ field (field at which $M$ reaches $0.95 M_{\mathrm{s}}$ ) of 4729.22 . In the case of sulphur substitution at $0.0 \%, H_{\mathrm{c}}$ was found to be $22.865, M_{\mathrm{s}} 14.127 \mathrm{E}-3, M_{\mathrm{r}} 80.666 \mathrm{E} 6$, and $H_{\mathrm{s}} 4704.80$ when compared with control, where coercive field $\left(H_{\mathrm{c}}\right)$ value is 10.973 , saturation magnetization $\left(M_{\mathrm{s}}\right)$ is $11.649 \mathrm{E}-3$, remanent magnetization $\left(M_{\mathrm{r}}\right)$ is $34.231 \mathrm{E}-6$ and saturation field $\left(H_{\mathrm{s}}\right)$, field at which $M$ reaches $0.95 M_{\mathrm{s}}$, is 4709.90, suggestive of enhanced magnetization ability after sulphur substitution (Fig. 6) (Rui and Shield 2006; Călin et al. 2011; Shah et al. 2015).

\section{Conclusion}

Soapnut extract was proved to be one of the best biological sources for the synthesis of sulphur-substituted $\mathrm{NdFeB}$ permanent magnetic materials through the adoption of a simple procedure with minimum energy consumption. Sulphur substitution against boron in $\mathrm{NdFeB}$ magnetic system has significant influence on surface morphological and magnetic properties. The substitution of sulphur at $0.3 \%$ against boron was proved to be a most successful concentration in achieving the significant multiple parameters including size, stability, and magnetization. The synthesized sulphur-substituted $\mathrm{NdFeB}$ ferromagnetic nanoparticles with enhanced magnetization could find versatile applications in different fields including medical diagnostics, non-chemotherapeutic cancer treatment and magnetic film coatings.

Acknowledgements GVSJRao is thankful to University Grants Commission (UGC) for funding under Faculty development programme (FDP) and Andhra University, Visakhapatnam and also thankful to Institute of Physics, Bhubaneswar and Indian Institute of Chemical Technology (IICT), Hyderabad and Indian Institute of Technology, Bhubaneswar for extending research facilities to carry out part of the characterization of the materials. Authors are thankful to the authorities of Acharya N G Ranga Agricultural University for providing research facilities at Institute of Frontier Technology, R.A.R.S., Tirupati to carry out part of research work.

Open Access This article is distributed under the terms of the Creative Commons Attribution 4.0 International License (http:// creativecommons.org/licenses/by/4.0/), which permits unrestricted use, distribution, and reproduction in any medium, provided you give appropriate credit to the original author(s) and the source, provide a link to the Creative Commons license, and indicate if changes were made.

\section{References}

Bharti A, Preethi B, Deepak T, Alok S (2012) Sapindus emarginatus: phytochemistry and various biological activities. Indo Global J Pharma Sci 2(3):250-257
Brown D, Ma BM, Chen Z (2002) Developments in the processing and properties of NdFeB-type permanent magnets. J Magn Magn Mater 248(3):432-440

Castaldi L, Davies HA, Gibbs MRJ (2002) Growth and characterization of NdFeB thin films. J Magn Magn Mater 242:1284-1286

Chopra N, Gavalas VG, Bachas LG, Hinds BJ, Bachas LG (2007) Functional one-dimensional nanomaterials: applications in nanoscale biosensors. Anal Lett 40(11):2067-2096

Călin M, Helerea E, Ritchie E (2011) Investigation procedure of magnetic performances of $\mathrm{NdFeB}$ permanent magnets. Bull Transilv Univ Brasov Ser I Eng Sci 4(2):99-106

Deheri PK, Swaminathan V, Bhame SD, Liu Z, Ramanujan RV (2010) Sol-gel based chemical synthesis of $\mathrm{Nd}_{2} \mathrm{Fe}_{14} \mathrm{~B}$ hard magnetic nanoparticles. Chem Mater 22(24):6509-6517

Elumalai EK, Prasad TNVKV, Nagajyothi PC, David E (2011) A bird's eye view on biogenic silver nanoparticles and their applications. Der Chem Sin. 2:88-97

George B, Shanmugam S (2014) Phytochemical screening and antimicrobial activity of fruits extract of Sapindus mukorossi. Int J Curr Microbiol Appl Sci 3:604-611

Gutfleisch O, Drazic G, Mishima C, Honkura Y (2002) Texture inducement during HDDR processing of NdFeB. IEEE Trans Magnet 38(5):2958-2960

Jadhav AP, Ma H, Kim DS, Baek YK, Choi CJ, Kang YS (2014) $\mathrm{Nd}_{2} \mathrm{Fe}_{14} \mathrm{~B}$ synthesis: effect of excess neodymium on phase purity and magnetic property. Bull Korean Chem Soc 35(3):886-890

Kotakadi VS, Gaddam SA, Rao YS, Prasad TN, Reddy AV, Gopal DS (2014) Biofabrication of silver nanoparticles using Andrographis paniculata. Eur J Med Chem 73:135-140

Liu Z, Zhao L (2017) Compositional optimization and new processes for nanocrystalline NdFeB-based permanent magnets. In: Zhang $\mathrm{S}$, Zhao D (eds) Advances in magnetic materials: processing, properties, and performance. CRC Press, Boca Raton, pp 293-372

Lu AH, Salabas EE, Schüth F (2007) Magnetic nanoparticles: synthesis, protection, functionalization, and application. Angew Chem Int Ed 46(8):1222-1244

Madhavi V, Prasad TNVKV, Madhavi G (2013) Synthesis and spectral characterization of iron based micro and nanoparticles. Int J Nanomater Biostruct 3(2):31-34

Prasad TNVKV, Elumalai EK, Khateeja S (2011) Evaluation of the antimicrobial efficacy of phytogenic silver nanoparticles. Asian Pac J Trop Biomed 1(1):S82-S85

Rao CN, Cheetham AK (2011) Science and technology of nanomaterials: current status and future prospects. J Mater Chem 11(12):2887-2894

Rui X, Shield JE (2006) In cluster-structured exchange-coupled magnets with high energy densities. Appl Phys Lett 89:122509

Shah IA, Abbas T, Ullah Z, Rauf A, Ullah K, Naseem S (2015) Sintering effects on structural and magnetic behaviours of NdFeB magnets. Armen J Phys 8(4):185-190

Sharma A, Sati SC, Sati OP, Sati D, Maneesha Kothiyal SK (2011) Chemical constituents and bioactivities of genus Sapindus. Int $\mathbf{J}$ Res Ayurveda Pharm 2(2):403-409

Sindhura KS, Prasad TNVKV, Selvam PP, Hussain OM (2014) Synthesis, characterization and evaluation of effect of phytogenic zinc nanoparticles on soil exo-enzymes. Appl Nanosci 4(7):819-827

Song JY, Jang HK, Kim BS (2009) Biological synthesis of gold nanoparticles using Magnolia kobus and Diopyros kaki leaf extracts. Process Biochem 44(10):1133-1138

Takahashi K, Kato H, Saito T, Matsuyama S, Kinugasa S (2008) Precise measurement of the size of nanoparticles by dynamic light scattering with uncertainty analysis. Part Part Syst Charact 25(1):31-38 
Thakkar JN, Mhatre SS, Parikh RY (2010) Biological synthesis of metallic nanoparticles. Nanomed Nanotechnol Biol Med 6(2):257-262

Tian MM, Chen DX, Sun YL, Yang YW, Jia Q (2013) Pillararenefunctionalized $\mathrm{Fe}_{3} \mathrm{O}_{4}$ nanoparticles as magnetic solid-phase extraction adsorbent for pesticide residue analysis in beverage samples. RSC Adv 3(44):22111-22119
Wu W, Wu Z, Yu T, Jiang C, Kim WS (2015) Recent progress on magnetic iron oxide nanoparticles: synthesis, surface functional strategies and biomedical applications. Sci Technol Adv Mater 16(2):023501

Zeng H, Sun S (2008) Syntheses, properties, and potential applications of multicomponent magnetic nanoparticles. Adv Funct Mater 18(3):391-400 